\section{How can nanosensors detect bacterial contamination before it ever reaches the dinner table?}
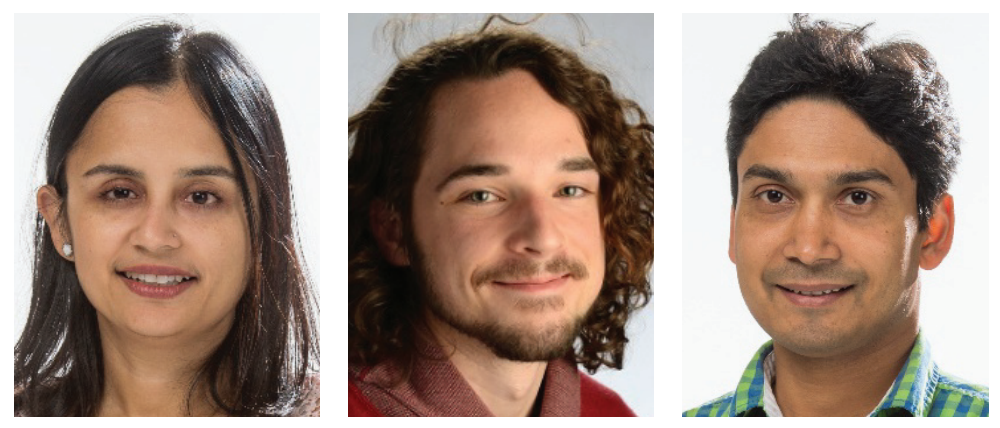

Tuhina Banerjee ${ }^{*, 1}$, Tyler Shelby ${ }^{1} \&$ Santimukul Santra**,1

First draft submitted: 10 October 2016; Accepted for publication: 26 October 2016; Published online: 20 January 2017

Most people like to know what they are getting when they order a meal or bring groceries home from the market, and an upset stomach is not the ideal follow-up to a tasty meal. A recent study by Centers for Disease Control and Prevention has shown that 31 major pathogens are responsible for around 10 million cases of foodborne illnesses annually in the USA alone [1]. Of these 10 million cases, roughly 56 thousand will require hospitalization, and nearly 1.4 thousand will result in death [2]. It is further estimated by the Centers for Disease Control and Prevention that when foodborne illnesses caused by unknown pathogenic agents are considered, the case total rises to roughly 48 million, and the death toll doubles to three thousand. Although there are many regulations in place to prevent the sale of contaminated foods and beverages, it is no simple task to ensure that each product remains pathogen free. Even without considering the recent rise of unregulated farmer's markets throughout the country, monitoring each and every commercially sold food product is an overwhelming task, and it is no wonder that foodborne illnesses are still so commonplace [3]. In fact, foodborne illnesses have recently become more common, despite advances in pathogen screening technology [4]. Within this editorial, it is our intention to discuss the most common causes of foodborne illnesses, methods by which contamination is traditionally detected and what current research has to offer, specifically in the field of nanoscience. In the future, it may be the case that food screening takes place in our own supermarkets or homes, and the groundwork for these possibilities will be outlined here.

Some of the most common pathogens associated with foodborne illness are Norovirus [5] and Salmonella spp [2]. Other pathogens that are notorious for ruining a good meal are Clostridium perfringens, Escherichia coli O157:H7, Campylobacter spp and Listeria ivanovii, among others $[2,6]$. Foodborne illnesses often result from consuming undercooked meat, or

\section{KEYWORDS}

- bacterial contamination

- foodborne illness • nanosensor

- pathogen detection

'Department of Chemistry, Kansas Polymer Research Center, Pittsburg State University, 1701 S Broadway Street, 
"In an effort to address the shortcomings of these detection techniques and design a more approachable and practical diagnostic platform, researchers from various fields of science are working to design more robust, timely and sensitive detection techniques.” ingesting food products that have come in contact with contaminated water sources or fecal matter during the processing stages [7]. Foods that require less processing, such as fresh fruits or vegetables, also are at greater risk for contamination [8]. Some classic methods used to detect pathogens include bacterial or viral culturing, PCR techniques and ELISA. Culturing of pathogens has long been favored in many cases due to its simplicity, cost-effectiveness and the fact that it results in a visible end-product: a living sample of the contaminant. However, this method is extremely inefficient with regard to time, and often takes multiple days or weeks for results to be produced. PCR and ELISA techniques are both steps forward with regard to timeliness, they still require hours if not days to complete, and are quite complex, requiring sample preparation, use of expensive and delicate enzymes, and access to top-of-the-line lab equipment [9]. In an effort to address the shortcomings of these detection techniques and design a more approachable and practical diagnostic platform, researchers from various fields of science are working to design more robust, timely and sensitive detection techniques. One field in which vast progress has been made is nanotechnology [10].

\section{How nanomaterials are changing the game}

Throughout the past decade there have been a number of exciting steps forward using novel nanotechnologies. Among these, modalities, such as magnetic resonance, fluorescence and colorimetric analysis have been used, in an effort to reduce the need for sample amplification and culturing time. Although these developing technologies vary in their mechanisms and designs, they all share a similar goal: timely detection of trace pathogen quantities.

Kaittanis et al. designed a method by which magnetic nanoparticles were able to specifically bind with and detect Mycobacterium avium (MAP) and cholera toxin [11,12]. MAP-specific antibodies were conjugated to the surface of superparamagnetic iron oxide nanoparticles for targeting. These conjugated nanoparticles were then able to sensitively bind to MAP in solution, and provide relaxation (T2) values using a magnetic relaxometer. Detection of MAP in quantities of 15.5 CFUs was possible within $30 \mathrm{~min}$, making this a remarkably fast and sensitive diagnostic platform. These assays were conducted in buffer as well as complex medium, such as milk and blood. Detection via this technique is much simpler than PCR or ELISA, as the only required steps for detection are the mixing of the nanoparticles with the collected samples and insertion of the mixture into the magnetic relaxometer. The nanoparticles are reportedly simple to synthesize and are stable for long periods of time, increasing their effectivity in a real-world setting.

Along similar lines, El-Boubbou et al. designed a diagnostic platform using magnetic glyconanoparticles which allowed for the detection of E. coli in $5 \mathrm{~min}$, and also provided a means to remove almost $90 \%$ of the $E$. coli from the solution [13]. This group functionalized silica-coated magnetite nanoparticles through surface conjugation of mammalian cell surface carbohydrates which allowed for binding to bacteria. Within $5 \mathrm{~min}$, these nanoparticle conjugates were able detect $E$. coli with a limit of $10^{4}$ cells $/ \mathrm{ml}$, and were able to capture $65 \%$ of the bacterial contaminants when a magnetic field was applied to the solution. If a longer incubation time was allowed ( $45 \mathrm{~min}$ ) then up to $88 \%$ of the contaminants would have been able to be sequestered by the magnetic nanoparticles. This unique approach provides a means by which contamination can not only be detected, but also may also be removed. While the carbohydrates conjugated to the surfaces of these nanoparticles did not allow for extreme specificity, there was a correlation between binding affinity and $E$. coli strain, which allowed the researchers to determine which specific strain of E. coli was in each solution.

Not all nanosensors rely on magnetic modalities, as shown by Miranda et al., who designed a colorimetric based detection technique using gold nanoparticles [14]. These gold nanoparticles were electrostatically bound to a deactivated enzyme. When bacteria were present, however, their interaction with the nanoparticle freed the enzyme and reactivated it. This reactivation was coupled with an enzyme-amplified colorimetric reading, allowing for visual detection of contaminants with a sensitivity threshold of $10^{2}$ bacteria $/ \mathrm{ml}$. Furthermore, this lab group designed a portable test strip capable of on-site detection of contamination with a threshold of $10^{4}$ bacteria $/ \mathrm{ml}$.

Similar research conducted by Bui et al., led to the production of a nanodiagnostic platform which also allowed for naked-eye detection of single-digit live bacteria [15]. This detection was visualized via a color shift from red to blue, which was caused by aggregation of plasmonic gold nanoparticles in the presence of bacteria. 
This aggregation is initially caused by a breakdown of cysteine-loaded nanoliposomes, triggered in the presence of single bacteria. Bacterial composites focused on in this work included Salmonella, Listeria and E. coli O157.

A combination of magnetic and colorimetric technology was used by Cho et al., [16]. In their research, magnetic nanoparticles were used to isolate bacterial contaminants which were then applied to an immunostrip, providing colorimetric data in the presence of contaminants. This technique allowed for successful detection within $2 \mathrm{~h}$ with sensitivity thresholds as little as $95 \pm 19.5 \mathrm{CFU} / \mathrm{ml}$.

Within the past year, Banerjee et al. designed a separate multimodal detection platform, featuring the combination of magnetic and fluorescence modalities [17]. Superparamagnetic iron oxide nanoparticles were loaded with fluorescent dye and then functionalized via surface conjugation with pathogen-specific antibodies. The unique pairing of these modalities allows for detection of contamination in a range of developments. Magnetic detection is able to produce reliable T2 shifts in early-stage pathogen contamination (1-25 CFU) and the fluorescence modality allows for the further characterization of more developed contamination ( $>25 \mathrm{CFU}$ ). This platform was able to detect E. coli O157:H7 contaminants within $30 \mathrm{~min}$ with sensitivity thresholds as little as $1 \mathrm{CFU}$.

\section{What the future holds}

In order for the discussed detection techniques to reach their full potential, they must eventually find their way out of the laboratory and into supermarkets, restaurants and even homes. Currently, commercial food may be screened for contamination at the site of production, but it must then pass between packaging facilities, transport vehicles, storage locations, vendor sites and finally homes before its journey ends. At each point in the process, food may come in contact with other products and cross-contamination is a possibility. Therefore, initial screening of food products does not ensure that pathogen-free produce will still be pathogen free when it enters your mouth. While many of the discussed techniques have advanced our ability to detect pathogens with regard to time and sensitivity, the techniques often still require large equipment. The future of this science relies on the adaptation of these detection modalities to a test-strip or chipstyled platform. A prototype of this technology was recently designed by Liong et al., who prepared a magnetic barcode assay which allowed for portable detection of Mycobacterium tuberculosis within $2.5 \mathrm{~h}$ [18]. Whether it is a salad or a stuffed burrito that catches your appetite, you may soon be able to scan your food yourself, ensuring that it will not bring any unpleasant surprises with it.

\section{Financial \& competing interests disclosure}

The authors have no relevant affiliations or financial involvement with any organization or entity with a financial interest in or financial conflict with the subject matter or materials discussed in the manuscript. This includes employment, consultancies, honoraria, stock ownership or options, expert testimony, grants or patents received or pending, or royalties.

No writing assistance was utilized in the production of this manuscript.

\author{
"The future of this science \\ relies on the adaptation of \\ these detection modalities \\ to a test-strip or chip-styled \\ platform."
}

\section{References}

1 Centers for Disease Control and Prevention. Estimates of Foodborne Illness in the United States.

www.cdc.gov/foodborneburden/index.html

2 Scallan E, Hoekstra RM, Angulo FJ et al. Foodborne illness acquired in the United States - major pathogens. Emerg. Infect. Dis. 17(1), 7-15 (2011).

3 Law JW, Ab Mutalib NS, Chan KG, Lee LH. Rapid methods for the detection of foodborne bacterial pathogens: principles, applications, advantages and limitations. Front. Microbiol. 5, 770 (2014).

4 Warriner K, Huber A, Namvar A, Fan W, Dunfield K. Recent advances in the microbial safety of fresh fruits and vegetables. Adv. Food Nutr. Res. 57 155-208 (2009).

5 Koo HL, Ajami N, Atmar RL, DuPont HL. Noroviruses: the leading cause of gastroenteritis worldwide. Discov. Med. 10(50), 61-70 (2010).

6 Alvarez-Ordóñez A, Leong D, Morgan CA, Hill C, Gahan CG, Jordan K. Occurrence, persistence, and virulence potential of Listeria ivanovii in foods and food processing environments in the Republic of Ireland. Biomed. Res. Int. 2015, 350526 (2015).

7 Mandal PK, Biswas AK, Choi K, Pal UK. Methods for rapid detection of foodborne pathogens: an overview. Am. J. Food Technol. 6(2), 87-102 (2011).
8 Jeddi MZ, Yunesian M, Gorji ME, Noori N, Pourmand MR, Khaniki GR. Microbial evaluation of fresh, minimally-processed vegetables and bagged sprouts from chain supermarkets. J.Health. Popul. Nutr. 32(3), 391-399 (2014).

9 Zhao X, Lin CW, Wang J, Oh DH. Advances in rapid detection methods for foodborne pathogens. J.Microbiol. Biotechnol. 24(3), 297-312 (2014).

10 Inbaraj BS, Chen BH. Nanomaterial-based sensors for detection of foodborne bacterial pathogens and toxins as well as pork adulteration in meat products. J. Food Drug. Anal. 24(1), 15-28 (2016).

11 Kaittanis C, Naser SA, Perez JM. One-step, nanoparticle-mediated bacterial detection 
EDITORIAL Banerjee, Shelby \& Santra

with magnetic relaxation. Nano Lett. 7(2), 380-383 (2007).

12 Kaittanis C, Banerjee T, Santra S, Santiesteban OJ, Teter K, Perez JM. Identification of molecular-mimicry-based ligands for cholera diagnostics using magnetic relaxation. Bioconjug. Chem. 22(2), 307-314 (2011).

13 El-Boubbou K, Gruden C, Huang X. Magnetic glyco-nanoparticles: a unique tool for rapid pathogen detection,

decontamination, and strain differentiation.
J. Am. Chem. Soc. 129(44), 13392-13393 (2007).

14 Miranda OR, Li X, Garcia-Gonzalez L et al. Colorimetric bacteria sensing using a supramolecular enzyme-nanoparticle biosensor. J. Am. Chem. Soc. 133(25), 9650-9653 (2011).

15 Bui MP, Ahmed S, Abbas A. Single-digit pathogen and attomolar detection with the naked eye using liposome-amplified plasmonic immunoassay. Nano Lett. 15(9), 6239-6246 (2015).
16 Cho IH, Irudayaraj J. Lateral-flow enzyme immunoconcentration for rapid detection of Listeria monocytogenes. Anal. Bioanal. Chem. 405(10), 3313-3319 (2013).

17 Banerjee T, Sulthana S, Shelby T et al. Multiparametric magneto-fluorescent nanosensors for the ultrasensitive detection of Escherichia coli O157:H7. ACS Infect. Dis. 2(10), 667-673 (2016).

18 Liong M, Hoang AN, Chung J et al. Magnetic barcode assay for genetic detection of pathogens. Nat. Commun. 4, 1752 (2013). 